\title{
Independent risk factors for axillary lymph node metastasis in breast cancer patients with one or two positive sentinel lymph nodes
}

Wei Zhang ${ }^{1}$, Jing $\mathrm{Xu}^{2}$, Ke Wang ${ }^{1}$, Xiao-Jiang Tang ${ }^{1}$, Hua Liang ${ }^{3}$ and Jian-Jun He ${ }^{1 *}$

\begin{abstract}
Background: The benefit of axillary lymph node dissection (ALND) in breast cancer patients with one or two positive sentinel lymph nodes (SLNs) remains inconclusive. The purpose of this study was to identify risk factors independently associated with axillary lymph node (ALN) metastasis.
\end{abstract}

Methods: We retrospectively analyzed data from 389 Chinese breast cancer patients with one or two positive SLNs who underwent ALND. Univariate and multivariate logistic regression analyses were performed to identify ALN metastasis-associated risk factors.

Results: Among the 389 patients, 174 (44.7\%) had ALN metastasis, while 215 (55.3\%) showed no evidence of ALN metastasis. Univariate analysis revealed significant differences in age ( $<60$ or $\geq 60$ years), human epidermal growth factor receptor-2 (Her-2) status, and the ratio of positive to total SLNs between the ALN metastasis and nonmetastasis groups $(P<0.05)$. The multivariate analysis indicated that age, the ratio of positive to total SLNs, and occupations were significantly different between the two groups. Lastly, younger age ( $<60$ years), a higher ratio of positive to total SLNs, and manual labor jobs were independently associated with ALN metastasis $(P<0.05)$.

Conclusions: The risk of ALN metastasis in breast cancer patients with one or two positive SLNs can be further increased by younger age, manual labor jobs, and a high ratio of positive to total SLNs. Our findings may also aid in identifying which patients with one or two positive SLNs may not require ALND.

Keywords: Breast cancer, Sentinel lymph node, Sentinel lymph node biopsy, Axillary lymph node, Axillary lymph node metastasis

\section{Background}

Breast cancer is the most common invasive malignancy detected in women around the globe, accounting for $22.9 \%$ of all female cancers [1-3]. In accordance to the World Health Organization (WHO), it has been estimated that 627,000 women died in 2018 from breast

\footnotetext{
* Correspondence: tougaohjj@126.com

1 Department of Breast Surgery, The First Affiliated Hospital of Xi'an Jiaotong University, 277 Yanta West Rd., Xi'an 710061, Shaanxi, China

Full list of author information is available at the end of the article
}

cancer worldwide, which is approximately $15 \%$ of cancer-related deaths in women [4]. Similar to other cancers, breast cancer cells can develop rapidly and become metastatic if left untreated or managed improperly in some cases, allowing cancer cells to spread to distant organs through nearby sentinel lymph nodes (SLNs), axillary lymph nodes (ALNs), or through other routes. The SLN is the node through which breast cancer cells spread from the original tumor site to the ALNs and other distant organs [5]. 
Sentinel lymph node biopsy (SLNB) was a landmark procedure that led to a fundamental shift in the management of the ALN metastases. In fact, SLNB has partly replaced the more traditional previous approach of axillary lymph node dissection (ALND) for the treatment of early-stage invasive breast cancer in some cases [6-14]. SLNB has also empowered physicians to assess disease stage, predict the prognosis of patients, and develop optimal treatment strategies for improving the quality of life and positive outcomes of patients $[6,10]$. When the SLNB yields a positive finding, standard practices recommend excision of the ALNs during the SLNB procedure or scheduled follow-up procedures [11]. The SLNB may also help some breast cancer patients avoid unnecessary and highly invasive lymph node surgeries, such as ALND, which is a procedure associated with serious complications [11-14]. In contrast to ALND, SLNB has a lower incidence of procedure-related complications, including shoulder stiffness, pain, and lymphedema. Furthermore, removal of nearby lymph nodes may be unnecessary when the SLNB result is negative [11-14]. As all lymph node surgeries have some degree of risk, adverse effects of the procedure may be minimized if fewer lymph nodes are removed.

It has been proposed that a surgical procedure on extensive lymph nodes, such as ALND, may be avoided in breast cancer patients with negative SLNB findings [1114]. Indeed, negative SLNB findings indicate that the breast cancer cells have not developed the ability to invade nearby lymph nodes in the body, while a positive SLNB result indicates that the cancer cells are present in the SLNs and may spread into nearby regional lymph nodes, and possibly distant organs of the body [5]. In addition, the value of ALND in breast cancer patients with one or two positive SLNs has been debated as omitting the procedure does not affect clinical outcomes [13]. In breast cancer patients with one SLN, the benefit of ALND has not been established. In addition, the incidence of ALN metastasis in breast cancer patients with one or two positive SLNs has not been largely studied in Chinese breast cancer patients. Therefore, it is important to identify risk factors associated with ALN status in breast cancer patients, particularly those with one or two positive SLNs who may or may not need immediate surgical intervention. Several previous studies indicated that a number of risk factors, such as age at the time of diagnosis, body mass index (BMI), tumor size, primary tumor quadrant, molecular subtype, estrogen receptor (ER) status, progesterone receptor (PR) status, human epidermal growth factor receptor-2 (Her-2) status, and other clinical parameters were associated with ALN metastasis in breast cancer [15-19]. However, the effects of these factors on the incidence of ALN metastasis were inconsistent due to ethnic/racial differences [19-23]. For this reason, researchers need a better understanding of the risk factors associated with ALN status, particularly in Chinese breast cancer patients with one or two positive SLNs.

In the present study, we retrospectively reviewed and analyzed data from Chinese breast cancer patients with one or two positive SLNs who had undertaken ALND with and without ALN metastasis. Univariate and multivariate logistic regression analyses were performed to determine the incidence of ALN metastasis in Chinese breast cancer patients with one or two positive SLNs and to identify risk factors independently associated with ALN metastasis. Our findings may allow predicting which patients are at high risk for ALN metastasis and may also lay the foundation for the development of an improved algorithm for predicting ALN status in breast cancer patients in the future.

\section{Methods}

\section{Human subjects and study design}

In this retrospective study, the demographic, social, laboratory tests, and clinical data of 389 Chinese breast cancer patients with one or two positive SLNs spanning the period between January 2010 to January 2018 at the First Affiliated Hospital of Xi'an Jiaotong University (Xi'an, Shaanxi, China) were reviewed and analyzed. The subjects who met the following inclusion criteria were consecutively enrolled in this study: (1) Primary cT1-2 N0 breast cancer was diagnosed and confirmed by pathological findings of a needle core biopsy; (2) presence of one or two positive SLNs; (3) ALND was performed. However, patients who had certain clinical conditions, such as bilateral breast cancer or metastasis to chest wall or other distant organs, were excluded from the study. From the 389 Chinese breast cancer subjects, 174 were diagnosed and confirmed to have ALN metastasis, while 215 showed no evidence of ALN metastasis. The study subjects were assigned to the two groups based on metastasis status with their demographic, social, laboratory tests, and clinical data. All patients in this study received post-surgery systemic chemotherapy in accordance with the local clinical practice guidelines.

The present study was approved by the Research Ethic Committee at the First Affiliated Hospital of Xi'an Jiaotong University (Xi'an, Shaanxi, China). Since this was a retrospective analysis of anonymous data, singed informed consent was waived by the committee. In addition, the study was carried out in compliance with the Declaration of Helsinki.

\section{Identification methods of SLNs}

During pathological evaluation of the SLN metastasis, the SLNs were detected and visualized with an injection 
of methylthioninium chloride (Jiangsu Jumpcan Pharmaceutical Co., Ltd., Shanghai, China), radioactive substances such as nano-carbon suspension (Chongqing Lummy Pharmaceutical Co., Ltd., Chongqing, China), or indocyanine green (Liaoning Tianyi Biopharmaceutical Limited by Share Ltd., Shenyang, China). The injected materials were directly visualized (blue dyes) or assessed by radioactive imaging. SLNs were examined the blue/ black-stained lymph vessels under the direction of a dye tracer. According to the manufacturer's protocol, an SLN was defined as any blue/black-stained lymph node, any lymph node with a blue/black-stained lymphatic channel which led directly to it, any lymph node with a radioactive count of $\geq 10 \%$ of the most radioactive lymph node, or any pathologically palpable lymph node. Fastfrozen pathology was conducted on each SLN to check for the presence of breast cancer cells. Two expert pathologists reviewed the pathological findings. ALND was performed if the fast-frozen pathology revealed SLN metastasis with one or two positive lymph nodes.

\section{Histological examination and immunohistochemistry}

All nodes were histologically examined with hematoxylin and eosin (H\&E) staining. The status of Her-2, ER, and PR were assessed by immunohistochemistry (IHC) in combination with fluorescence in situ hybridization (FISH). The Her-2 positivity was defined as a score of $3+$ alone or $\leq 2+$ when combined with fluorescence in situ hybridization (FISH) findings [24-26].

\section{Univariate logistic regression analysis}

The demographic, social, and clinicopathologic parameters, as well as laboratory tests findings, were used for the univariate logistic regression analysis to identify ALN metastasis-associated risk factors in Chinese breast cancer patients with one or two positive SLNs. For the univariate logistic regression analysis, the following demographic and social factors were included: age $(<60$ or $\geq 60$ ) and occupations, in which the study patients were categorized by occupational types into three groups: manual labor jobs (blue-color jobs with tasks requiring muscle power), mental labor jobs (white-color jobs such as teachers, librarians, and administrative jobs), and the other/rest (e.g. housekeeper, freelancer). In addition, the clinical, pathological, and laboratory tests parameters, which included menarche, menopausal status (pre-menopausal, post-menopausal as determined at the time of the initial diagnosis), delay in diagnosis (0-90, 91-180, 181-365, or $\geq 366$ days), tumor primary site (right or left), tumor location (upper outer, lower outer, upper inner, or lower inner), tumor size $(\leq 2 \mathrm{~cm}$ or $>2 \mathrm{~cm}$ ), pathological type [invasive ductal carcinoma (IDC) or other], ER status (negative or positive), PR status (negative or positive), Her-2 status (negative or positive), triple negative status (yes or no), Ki-67 status (high or low based upon the cut-off value of 30\%), tumor grade $(1,2$, or 3$)$, postoperative ALNs $(\leq 10,11-19$, or $\geq$ 20) upon postoperative pathological examination, and the ratio of positive to total SLNs $(\leq 0.05$ or $>0.5)$ were integrated into the univariate logistic regression analysis to determine which of those were associated with the status of ALN metastasis in breast cancer patients with one or two positive SLNs.

\section{Multivariate logistic regression analysis}

The demographic, social, and clinicopathologic characteristics were also subjected to a multivariate logistic regression analysis to determine independent risk factors for prediction of ALN metastasis in breast cancer patients with one or two positive SLNs. In addition, odds ratios (OR) with 95\% confidence interval (CI) were calculated and presented.

\section{Statistical analysis}

In this study, all variables except for age were categorical variables. Age was converted to a categorical variable for analysis. Categorical data were presented as frequencies and percentages. The Pearson's chi-squared test $\left(\chi^{2}\right)$ was used to assess differences in axillary lymph node metastasis negative and positive group (negative vs. positive). The likelihood ratio and Pearson's statistics were used to evaluate differences in the contingency tables. Univariate logistic regression was performed to evaluate the independent correlation between potential risk factors and ALN metastasis in breast cancer patients with one or two positive SLNs. Multivariate proportional hazard analyses were performed to select major significant variables, and the selection of variables was based on the forward stepwise method using a $P$-value threshold. The multivariate logistic analysis was used to create logistic models. OR and corresponding 95\% CI were estimated for each factor. A $p$-value of $<0.05$ (two-sided) was considered to be statistically significant. All statistical analyses were conducted using SAS 9.4 (SAS Institute, Inc., Cary, NC, USA). Mathematica 10.1 (Wolfram Research Inc., Champaign, IL, USA) was utilized to calculate risk values.

\section{Results \\ Demographic and clinical characteristics of the study subjects}

A total of 389 breast cancer patients who had one or two positive SLNs and fulfilled the eligibility criteria were retrospectively analyzed. Of the 389 cases, 174 cases were pathologically diagnosed and confirmed to have breast cancer with ALN metastasis (44.7\%), while 215 (55.3\%) individuals had no signs of ALN metastasis. The detailed demographic and clinicopathologic 
characteristics, as well as laboratory tests findings, are summarized in Table 1 . The average age of the patients was 51 years and ranged from 24 to 85 years. The two groups exhibited significant differences in age distribution, Her-2 status, and ratio of positive to total SLNs $(P<0.05)$, whereas the remaining characteristics including delay in diagnosis, tumor primary site, tumor location, tumor size, pathological type, ER status, PR status, triple negative status, Ki-67 status, tumor grade, and postoperative ALNs were not significantly different between the two groups $(P>0.05)$.

\section{Univariate logistic regression analysis of the risk factors associated with ALN metastasis}

Univariate logistic regression analysis of the demographic and clinicopathologic parameters was used to explore ALN metastasis-associated risk factors in breast cancer patients with one or two positive SLNs (Table 2). The patient's age, occupation, and the ratio of positive to total SLNs were found to be strongly associated with ALN metastasis $(P<0.05)$, whereas ALN metastasis was not associated with the delay in diagnosis, tumor primary site, tumor location, tumor size, pathological type, ER status, PR status, Her-2 status, triple negative status, Ki-67 status, tumor grade, or postoperative ALNs $(P>0.05)$.

\section{Multivariate logistic regression analysis of risk factors associated with ALN metastasis in breast cancer patients with one or two positive SLNs}

The demographic, social, and clinicopathological characteristics, as listed in Table 1, were also used for the multivariate logistic regression analysis to determine independent risk factors for prediction of ALN metastasis in the breast cancer patients with one or two positive SLNs. The resulting data were expressed as ORs with 95\% CIs. As shown in Table 3, age (OR 0.612, 95\% CI: $0.379-0.989$ ), the ratio of positive to total SLNs (OR 2.603, 95\% CI: $1.765-3.838$ ), and occupation (OR 0.786, 95\% CI: 0.626-0.985) were found to be significantly different between the two groups (positive versus negative ALN metastasis), and these risk factors were independently associated with ALN metastasis in breast cancer patients with one or two positive SLNs $(P<0.05)$.

\section{Discussion}

Breast cancer patients who undergo the ALND procedure often experience serious complications associated with the surgery. To date, it is still debated if ALND should be avoided in breast cancer patients with one or two positive SLNs [13, 26-29]. Currently, the recommendation often relies on whether the patient presents with ALN metastasis. Until now, the incidence of ALN metastasis in breast cancer patients with one or two positive SLNs has not been largely investigated and the benefit of ALND has not been established in Chinese breast cancer patients with one or two positive SLNs.

In this study, we evaluated Chinese breast cancer patients with one or two positive SLNs who undergone ALND with or without ALN metastasis. There were four primary findings from this study. (1) Of the breast cancer patients with one or two positive SLNs, a smaller proportion of the study subjects had ALN metastasis (Table 1); (2) univariate logistic regression analysis showed that age, Her-2 status, and the ratio of positive to total SLNs were significantly associated with ALN metastasis (Table 2); (3) multivariate logistic regression analysis showed that age, occupation, and ratio of positive to total SLNs differed significantly between patients with and without ALN metastasis, suggesting that they were independently associated with ALN metastasis (Table 3); and (4) breast cancer patients with one or two positive SLNs who were 60 years of age or older, performed mental labor jobs, and had a lower ratio of positive to total SLNs showed low likelihood for developing ALN metastasis, allowing for the ALND procedure to be omitted from the treatment strategy. However, the risk for positive ALN was higher in breast cancer patients with one or two positive SLNs who were less than 60 years of age, performed manual labor jobs, and had a higher ratio of positive to total SLNs. In these patients, ALND was recommended for improved clinical outcomes.

The present study demonstrated that ALN metastasis was less prevalent in breast cancer patients with one or two SLNs in older women ( $\geq 60$ years of age) than younger women $(<60$ years of age). It has been welldocumented that older age is a risk factor for many forms of cancer, including breast cancer $[3,30]$, with approximately $40 \%$ of breast cancer cases occurring in older women [30]. However, the breast tissues of older women show age-associated changes in the sensitivity to female hormones and often present with favorable histology and low-grade disease [31]. This alteration in estrogen sensitivity and other biological changes, such as tumor microenvironment modifications, immune senescence, and epithelial cells may be contributing factors [31]. In this study, we discovered that occupation types [manual labor jobs, mental labor jobs, and others (the rest)] affected the status of ALN metastasis as patients who performed manual labor jobs showed a higher incidence of ALN metastasis than those who performed mental work or other occupations (the rest). In addition, we identified the ratio of positive to all SLNs as an independent risk factor for ALN metastasis, as it was positively associated with a higher risk for ALN metastasis in breast cancer patient with one or two positive SLNs.

In this retrospective study, a proportion of the study patients showed a positive family history of breast cancer or other cancers in their first-degree relatives (FDRs: 
Table 1 Sociodemographic and clinical characteristics of the study subjects

\begin{tabular}{|c|c|c|c|c|c|}
\hline \multicolumn{2}{|l|}{$\mathrm{N}(\%)$} & \multicolumn{4}{|c|}{ Patients with one or two SLNs $(n=389)$} \\
\hline Variable & Characteristics & Negative ALN $(n=215)$ & Positive ALN $(n=174)$ & $x^{2} / Z$ & $P$ \\
\hline \multicolumn{6}{|c|}{ Demographic } \\
\hline \multirow[t]{3}{*}{$\mathrm{X} 1$} & Age & & & & \\
\hline & $<60$ & $162(75.3)$ & $146(83.9)$ & 4.2734 & 0.0387 \\
\hline & $\geq 60$ & $53(24.7)$ & $28(16.1)$ & & \\
\hline \multirow[t]{4}{*}{$x 2$} & Occupation & & & & \\
\hline & Manual labor & $96(44.7)$ & $95(54.6)$ & 4.9281 & 0.0851 \\
\hline & Mental work & $51(23.7)$ & $40(23.0)$ & & \\
\hline & Other & $68(31.6)$ & $39(22.4)$ & & \\
\hline \multirow[t]{3}{*}{ X3 } & Menarche $^{a}$ & & & & \\
\hline & $<15$ & $148(68.8)$ & $110(63.2)$ & 1.6259 & 0.2023 \\
\hline & $\geq 15$ & $52(0.24)$ & $52(29.9)$ & & \\
\hline \multirow[t]{3}{*}{ X4 } & Menopausal status & & & & \\
\hline & Premenopausal & $121(56.3)$ & $98(56.3)$ & 0.0001 & 0.9933 \\
\hline & postmenopausal & $94(43.7)$ & $76(43.7)$ & & \\
\hline \multicolumn{6}{|c|}{ Clinical Characteristics } \\
\hline \multirow[t]{5}{*}{$\times 5$} & Delay in diagnosis (days) ${ }^{a}$ & & & & \\
\hline & $0-90$ & $86(40.0)$ & $68(39.1)$ & 0.0168 & 0.9866 \\
\hline & $91-180$ & $49(22.8)$ & $39(22.4)$ & & \\
\hline & $181-365$ & $28(13.0)$ & $29(16.7)$ & & \\
\hline & $>366$ & $51(23.7)$ & $37(21.3)$ & & \\
\hline \multirow[t]{3}{*}{ X6 } & Tumor primary site & & & & \\
\hline & Right & $119(55.3)$ & $80(46.0)$ & 3.3805 & 0.066 \\
\hline & Left & $96(44.7)$ & $94(54.0)$ & & \\
\hline \multirow[t]{5}{*}{ X7 } & Tumor location ${ }^{a}$ & & & & \\
\hline & Upper Outer & $125(58.1)$ & $113(64.9)$ & 5.2315 & 0.1556 \\
\hline & Lower Outer & $34(15.8)$ & $30(17.2)$ & & \\
\hline & Upper Inner & $41(19.1)$ & $19(10.9)$ & & \\
\hline & Lower Inner & $12(5.6)$ & $12(6.9)$ & & \\
\hline \multirow[t]{3}{*}{$x_{8}$} & Tumor Size $^{a}$ & & & & \\
\hline & $\leq 2 \mathrm{~cm}$ & $89(41.4)$ & $75(43.1)$ & 0.0299 & 0.8626 \\
\hline & $>2 \mathrm{~cm}$ & $112(52.1)$ & $91(52.3)$ & & \\
\hline \multirow[t]{3}{*}{ X9 } & Pathological type ${ }^{a}$ & & & & \\
\hline & IDC & 199 (92.6) & $159(91.4)$ & 0.1822 & 0.6695 \\
\hline & Other & $16(74.4)$ & $15(8.62)$ & & \\
\hline \multirow[t]{3}{*}{$\times 10$} & $E R^{a}$ & & & & \\
\hline & $(-)$ & $42(19.5)$ & $36(20.7)$ & 0.0614 & 0.8043 \\
\hline & $(+)$ & $169(78.6)$ & $136(78.2)$ & & \\
\hline \multirow[t]{3}{*}{$X 11$} & $\mathrm{PR}^{\mathrm{a}}$ & & & & \\
\hline & $(-)$ & $66(30.7)$ & $55(31.6)$ & 0.0213 & 0.8839 \\
\hline & $(+)$ & $145(67.4)$ & $117(67.2)$ & & \\
\hline \multirow[t]{3}{*}{$X 12$} & HER-2a & & & & \\
\hline & $(-)$ & 137 & 106 & 0.0303 & 0.8618 \\
\hline & $(+)$ & 38 & 28 & & \\
\hline
\end{tabular}


Table 1 Sociodemographic and clinical characteristics of the study subjects (Continued)

\begin{tabular}{|c|c|c|c|c|c|}
\hline \multicolumn{2}{|l|}{$\mathrm{N}(\%)$} & \multicolumn{4}{|c|}{ Patients with one or two SLNs $(n=389)$} \\
\hline Variable & Characteristics & Negative ALN $(n=215)$ & Positive ALN $(n=174)$ & $x^{2} / Z$ & $P$ \\
\hline \multirow[t]{3}{*}{$\mathrm{X} 13$} & Triple negative $^{a}$ & & & & \\
\hline & Yes & $15(6.98)$ & $14(8.00)$ & 0.1933 & 0.6601 \\
\hline & No & $188(87.4)$ & $148(85.1)$ & & \\
\hline \multirow[t]{3}{*}{$\mathrm{X} 14$} & $\mathrm{Ki}-67^{\mathrm{a}}$ & & & & \\
\hline & (low) & $164(76.3)$ & $133(76.4)$ & 0.0521 & 0.8195 \\
\hline & (high) & $47(21.9)$ & $36(20.7)$ & & \\
\hline \multirow[t]{4}{*}{$\mathrm{X} 15$} & Tumor Grade ${ }^{a}$ & & & & \\
\hline & 1 & $28(13.0)$ & $18(10.3)$ & 0.5895 & 0.5555 \\
\hline & 2 & $73(34.0)$ & $59(33.9)$ & & \\
\hline & 3 & $114(53.0)$ & $95(54.6)$ & & \\
\hline \multirow[t]{4}{*}{ X16 } & Postoperative $\mathrm{ALNs}^{\mathrm{a}}$ & & & & \\
\hline & $0-10$ & $55(25.6)$ & $53(30.5)$ & -0.0294 & 0.9765 \\
\hline & $11-19$ & $136(63.3)$ & $94(54.0)$ & & \\
\hline & $\geq 20$ & $19(8.8)$ & $25(14.4)$ & & \\
\hline \multirow[t]{3}{*}{$\mathrm{X} 17$} & Ratio of positive to total SLNs ${ }^{a}$ & & & & \\
\hline & $<0.5$ & $155(72.1)$ & $86(49.4)$ & 20.4071 & $<0.0001$ \\
\hline & $\geq 0.5$ & $60(28.0)$ & $87(50.0)$ & & \\
\hline
\end{tabular}

Abbreviations: $E R$ estrogen receptor, $P R$ progesterone receptor, Her-2 human epidermal growth factor receptor-2, $S L N$ sentinel lymph node, $A L N$ axillary lymph node, IDC invasive ductal carcinoma, SD standard deviation. ${ }^{\mathrm{a}}$ Missing data: Menarche $(n=27)$; Delay in diagnosis (days) ( $\left.\mathrm{n}=2\right)$; Tumor primary quadrant $(n=3) ;$ estrogen receptor (ER) ( $n=6)$; Progesterone receptor (PR) $(n=6)$, Her-2 $(n=12)$; Ki-67 $(n=8)$; Tumor size $(n=22)$; Triple negative $(n=24)$; Tumor grade $(n=2)$; Ratio of positive to total SLNs $(n=1)$; and postoperative ALNs $(n=3)$. All lymph nodes were examined with hematoxylin and eosin $(\mathrm{H} \& \mathrm{E})$ staining. Her-2 positivity was defined as a score of $3+$ or $\leq 2+$ combined with an application on fluorescence in situ hybridization (FISH). The molecular subtypes were determined according to the criteria by the World Health Organization

Table 2 Univariate logistic regression analysis of the relationship between sociodemographic, clinical, and pathological factors and ALN metastasis in breast cancer patients with one or two positive SLNS

\begin{tabular}{|c|c|c|c|c|c|c|}
\hline & Parameter & $\beta$ & SE & $P$ & OR & $95 \% \mathrm{Cl}$ \\
\hline$\overline{X 1}$ & Age & -0.5341 & 0.26 & 0.04 & 0.586 & $0.352-0.976$ \\
\hline $\mathrm{X} 2$ & Occupation & -0.2695 & 0.1221 & 0.0273 & 0.764 & $0.601-0.970$ \\
\hline X3 & Menarche & 0.2967 & 0.233 & 0.2029 & 1.345 & $0.852-2.124$ \\
\hline$X 4$ & Menopausal status & -0.00174 & 0.2056 & 0.9933 & 0.998 & $0.667-1.494$ \\
\hline$\times 5$ & Delay in diagnosis (days) & -0.00233 & 0.086 & 0.9784 & 0.998 & $0.843-1.181$ \\
\hline$x_{6}$ & Tumor primary site & 0.3760 & 0.2048 & 0.0664 & 1.457 & $0.975-2.176$ \\
\hline $\mathrm{X} 7$ & Tumor primary quadrant & -0.1329 & 0.1088 & 0.2218 & 0.876 & $0.707-1.084$ \\
\hline$x_{8}$ & Tumor Size & -0.0365 & 0.2109 & 0.8626 & 0.964 & $0.638-1.458$ \\
\hline$\times 9$ & Pathology & 0.1599 & 0.3748 & 0.6697 & 1.173 & $0.563-2.446$ \\
\hline $\mathrm{X} 10$ & ER & -0.0632 & 0.2547 & 0.804 & 0.939 & $0.570-1.546$ \\
\hline $\mathrm{X} 11$ & $P R$ & -0.0323 & 0.2209 & 0.8839 & 0.968 & $0.628-1.493$ \\
\hline $\mathrm{X} 12$ & HER2 & -0.0487 & 0.2806 & 0.8621 & 0.952 & $0.549-1.651$ \\
\hline $\mathrm{X} 13$ & Triple negative & -0.1542 & 0.3865 & 0.69 & 0.857 & $0.402-1.828$ \\
\hline $\mathrm{X} 14$ & Ki-67 & -0.0570 & 0.2503 & 0.8199 & 0.945 & $0.578-1.543$ \\
\hline $\mathrm{X} 15$ & Tumor grade & 0.0994 & 0.1481 & 0.502 & 1.105 & $0.826-1.476$ \\
\hline $\mathrm{X} 16$ & Postoperative ALNs & -0.0632 & 0.089 & 0.4778 & 0.939 & $0.789-1.118$ \\
\hline $\mathrm{X} 17$ & Ratio of positive to total SLNs & 0.9606 & 0.215 & $<0.0001$ & 2.613 & $1.715-3.983$ \\
\hline
\end{tabular}

Abbreviations: $O R$ odds ratio, confidential interval, $E R$ estrogen receptor, $P R$ progesterone receptor, Her-2 human epidermal growth factor receptor-2, SLN sentinel lymph node, $A L N$ axillary lymph node. To avoid missing any significant indicators, the factors with a significance of $P<0.05$ in the univariate logistic regression analysis, including age, occupation, and the ratio of positive to the total number of SLNs were considered to entry into the multivariate logistic regression analysis 
Table 3 Multivariable logistic regression analyses of the risk factors associated with ALN metastasis in breast cancer patients with one or two positive SLNS

\begin{tabular}{|c|c|c|c|c|c|c|}
\hline & Parameter & $\beta$ & SE & $P$ & OR & $95 \% \mathrm{Cl}$ \\
\hline & Intercept & -0.5262 & 0.4468 & 0.239 & & \\
\hline$\overline{x 1}$ & Age & -0.4902 & 0.2447 & 0.0451 & 0.612 & $0.379-0.989$ \\
\hline$\times 2$ & Occupation & -0.2414 & 0.1155 & 0.0365 & 0.786 & $0.626-0.985$ \\
\hline $\mathrm{X} 17$ & Ratio of positive to total SLNs & 0.9565 & 0.1982 & $<0.0001$ & 2.603 & $1.765-3.838$ \\
\hline
\end{tabular}

father, mother, sisters, or brothers). However, there was no significant difference between the ALN metastasis and non-metastasis groups.

In this study, we emphasized a Chinese patient cohort primarily because differences exist between Chinese and Western populations. Several previous studies suggested that the risk factors for breast cancer had differential effects on the ALN metastasis among the different populations. Çetintaş and colleagues have reported that other independent risk factors, including the presence of perineural invasion, lymphatic vessel invasion, age of $<40$ years, and an extensive intraductal component ( $>25 \%$ ), were significantly associated with an increased risk of ALD metastasis in breast cancer patients [32]. In particular, Liu and colleagues reported distinct tumor characteristics between Chinese and Western breast cancer patients, including that the number of isolated tumor cells (ITCs) and micrometastases in Chinese breast cancer patients was much lower compared to Western breast cancer patients [33]. Until now, the risk factors were identified, and diagnostic nomograms were developed mainly in the Western population. Considering these differences, we strongly believe that there is a need for risk factors to be investigated in Chinese women by applying their own sociodemographic factors to better represent the risk factors associated with ALN status in Chinese breast cancer patients with one or two positive SLNs.

In this study, the occupation was categorized into manual and mental labor jobs. In contrast to mental labor jobs, responsibilities in manual or physical labor jobs involve more physical or laborious tasks using their muscle power. Previous studies on occupation and risk of breast cancer have indicated variations in the risk of developing breast cancer between two occupational groups: white-collar and blue-collar females [34-36]. White-collar women who worked as teachers, librarians, or administrative jobs were at a higher risk of developing breast cancer than blue-collar women [34-36]. The causes underlying this difference are unknown, yet it has been speculated that late first-time pregnancy might contribute to the difference. In the present study, to assess an association between occupation and the incidence of ALN metastases, occupation was categorized into manual and mental labor jobs, and the study patients were subdivided into three subgroups: manual labor jobs, mental labor jobs, and others/rest.

Under the presence of the three independent factors, including younger age ( $<60$ years), manual worker, a higher ratio of positive to total SLNs, patients experienced an increased risk for development of ALN metastasis. Oppositely, older age ( $\geq 60$ years), mental labor jobs, and a lower ratio of positive to total SLNs were indicative of a lower likelihood of ALN metastasis, which meant that ALND could be unnecessary or could have been avoided in these breast cancer patients. Thus, the findings of this study may aid in the treatment decisionmaking process, leading to better clinical outcomes. In this study, we integrated a delay in the diagnosis of breast cancer into both univariate and multivariate logistic regression analyses, as the diagnosis delay with the time between the onset of symptoms and the initial medical consultation, professional delay between the initial consultation and the initial treatment, and between the time from the initial onset of symptoms to the initial treatment were previously found to be associated with the prognosis of cancer patients [37, 38]. In our study, various time periods in the diagnosis delay were divided into four categories, including 0-90, 91-180, 181-365, and $\geq 366$ days, which were used in the univariate and multivariate logistic regression analyses. The resulting data did not exhibit any significant correlations between the delay in the diagnosis of breast cancer and the status of ALN metastasis.

The American College of Surgeons Oncology Group (ACOSOG) Z0011 trial has shown that ALND offers no additional benefit and may not be necessary in some breast cancer patients, suggesting the change of clinical ALN surgery management for eligible breast cancer patients. We noticed that the Z0011 clinical trial had specific criteria (e.g., $\mathrm{T} \leq 5 \mathrm{~cm}$, breast-conserving surgery, SLNs $\leq 2$, postoperative radiotherapy) for omission of ALND, which would not be feasible for breast cancer patients who did not meet the criteria. Therefore, the clinical significance of the Z0011 study remains controversial. There is still debate about the most appropriate approach to implement the findings of the Z0011 trial into clinical practice, and the benefit of ALND in breast cancer patients with 1-2 positive SLNs remains inconclusive in the post- 
ACOSOG Z0011 trial era. Our study has identified independent risk factors for ALN metastasis in Chinese breast cancer patients with 1-2 positive SLNs, which allow for predicting the patients at low risk for ALN metastasis, who may not require ALND. Considering that in most centers in China, including ours, the current treatment remains ALND in breast cancer patients with positive SLNs, our results may assist clinicians in implementing or omitting ALND in clinical practice.

We realized that the present study has limitations. For example, this study was conducted using the data of breast cancer patients from a single hospital, yet prospective studies at multiple centers with different titers would better represent the entire population of breast cancer patients. We also realized that the small number of patients may have affected the statistical power of this study, and future studies should recruit larger patient populations. In addition, future studies should construct a predictive nomogram based on the multivariate logistic regression results, allowing them to externally validate the reliability and reproducibility of these findings. There are ongoing relevant studies in our center.

\section{Conclusions}

In summary, the results of this retrospective study have suggested that age, the ratio of positive to total SLNs, and occupation are independent risk factors associated with the status of ALN metastasis in breast cancer patients with one or two SLNs. Our findings also suggest that, in older breast cancer patients ( $\geq 60$ years) who performed mental labor jobs and had a lower ratio of positive to total SLNs $(<0.5)$, the likelihood of ALN metastasis was relatively low. In contrast, breast cancer patients who were younger ( $<60$ years of age), performed manual labor jobs, and had a higher ratio of positive to total SLNs could be at relatively high risk for ALN metastasis and the ALND procedure could be recommended in these patients to improve their overall survival.

\footnotetext{
Abbreviations

ACOSOG: American College of Surgeons Oncology Group; ALND: Axillary lymph node dissection; ALNs: Axillary lymph nodes; BMI: Body mass index; Cl: Confidence interval; ER: Estrogen receptor; FISH: Fluorescence in situ hybridization; Her-2: Human epidermal growth factor receptor-2; H\&E: Hematoxylin and eosin; IDC: Invasive ductal carcinoma; IHC: Immunohistochemistry; IRB: Institutional Review Board; ITCs: Isolated tumor cells; OR: Odds ratio; PR: Progesterone receptor; SD: Standard deviation; SLNs: Sentinel lymph nodes; SLNB: Sentinel lymph node biopsy; WHO: World Health Organization
}

\section{Acknowledgments}

None.

\section{Authors' contributions}

WZ and JJH designed the study. WZ, JX, KW, XJT and JJH collected and analyzed the data. WZ, JX and KW drafted and wrote the manuscript. JJH and $\mathrm{HL}$ revised the manuscript critically for intellectual content. All authors gave intellectual input to the study and approved the final version of the manuscript.

\section{Funding}

This study was supported by the Key Research and Development Plan-Social Development Area of Shaanxi Province (No. 2018SF_156), and the research grant of the First Affiliated Hospital of Xi'an Jiaotong University (No. 2017QN-

16). The authors declare that the funding body was not involved in study design, data collection, analysis, interpretation and writing of the study.

\section{Availability of data and materials}

The datasets generated and analyzed during the current study are available from the corresponding author on reasonable request.

\section{Ethics approval and consent to participate}

The present study was approved by the Research Ethic Committee at the First Affiliated Hospital of Xi'an Jiaotong University (Xi'an, Shaanxi, China). Since this study was a retrospective analysis of anonymous data, signed informed consent to participate was waived by the committee.

\section{Consent for publication}

Not Applicable.

\section{Competing interests}

The authors declare that they have no competing interests.

\section{Author details}

${ }^{1}$ Department of Breast Surgery, The First Affiliated Hospital of Xi'an Jiaotong University, 277 Yanta West Rd., Xi'an 710061, Shaanxi, China. ${ }^{2}$ Department of Geriatric Medicine, The First Affiliated Hospital of Xi'an Jiaotong University, Xi'an 710061, Shaanxi, China. ${ }^{3}$ Department of Pathology, The First Affiliated Hospital of Xi'an Jiaotong University, Xi'an 710061, Shaanxi, China.

Received: 21 August 2019 Accepted: 26 June 2020

Published online: 09 July 2020

\section{References}

1. McGuire A, Brown JA, Malone C, McLaughlin R, Kerin MJ. Effects of age on the detection and management of breast cancer. Cancers (Basel). 2015;7: 908-29.

2. Torre LA, Bray F, Siegel RL, Ferlay J, Lortet-Tieulent J, Jemal A. Global cancer statistics, 2012. CA Cancer J Clin. 2015;65:87-108.

3. Siegel RL, Miller KD, Jemal A. Cancer statistics, 2016. CA Cancer J Clin. 2016; 66:7-30.

4. Bray F, Ferlay J, Soerjomataram I, Siegel RL, Torre LA, Jemal A. Global cancer statistics 2018: GLOBOCAN estimates of incidence and mortality worldwide for 36 cancers in 185 countries. CA Cancer J Clin. 2018;68:394-424.

5. Apple SK. Sentinel lymph node in breast Cancer: review article from a Pathologist's point of view. J Pathol Transl Med. 2016;50:83-95.

6. Mansel RE, Fallowfield L, Kissin M, Goyal A, Newcombe RG, Dixon JM, et al. Randomized multicenter trial of sentinel node biopsy versus standard axillary treatment in operable breast cancer: the ALMANAC trial. J Natl Cancer Inst. 2006:98:599-609.

7. Fleissig A, Fallowfield LJ, Langridge Cl, Johnson L, Newcombe RG, Dixon JM et al. Post-operative arm morbidity and quality of life. Results of the ALMANAC randomised trial comparing sentinel node biopsy with standard axillary treatment in the management of patients with early breast cancer. Breast Cancer Res Treat. 2006;95:279-93.

8. Del Bianco P, Zavagno G, Burelli P, Scalco G, Barutta L, Carraro P, et al. Morbidity comparison of sentinel lymph node biopsy versus conventional axillary lymph node dissection for breast cancer patients: results of the sentinella-GIVOM Italian randomised clinical trial. Eur J Surg Oncol. 2008;34: 508-13.

9. Krag DN, Anderson SJ, Julian TB, Brown AM, Harlow SP, Ashikaga T, et al Technical outcomes of sentinel-lymph-node resection and conventional axillary-lymph-node dissection in patients with clinically node-negative breast cancer: results from the NSABP B-32 randomised phase III trial. Lancet Oncol. 2007:8:881-8.

10. Purushotham AD, Upponi S, Klevesath MB, Bobrow L, Millar K, Myles JP, et al. Morbidity after sentinel lymph node biopsy in primary breast cancer: results from a randomized controlled trial. J Clin Oncol. 2005;23:4312-21. 
11. Lyman GH, Giuliano AE, Somerfield MR, Benson AB 3rd, Bodurka DC, Burstein $\mathrm{HJ}$, et al. American Society of Clinical Oncology guideline recommendations for sentinel lymph node biopsy in early-stage breast cancer. J Clin Oncol. 2005;23:7703-20.

12. Veronesi U, Paganelli G, Viale G, Luini A, Zurrida S, Galimberti V, et al. A randomized comparison of sentinel-node biopsy with routine axillary dissection in breast cancer. N Engl J Med. 2003;349:546-53.

13. Giuliano AE, Hunt KK, Ballman KV, Beitsch PD, Whitworth PW, Blumencranz PW, et al. Axillary dissection vs no axillary dissection in women with invasive breast cancer and sentinel node metastasis: a randomized clinical trial. JAMA. 2011;305:569-75.

14. Giuliano AE, Ballman K, McCall L, Beitsch P, Whitworth PW, Blumencranz P, et al. Locoregional recurrence after sentinel lymph node dissection with or without axillary dissection in patients with sentinel lymph node metastases: long-term follow-up from the American College of Surgeons oncology group (Alliance) ACOSOG Z0011 randomized trial. Ann Surg. 2016;264: 413-20.

15. Viale G, Zurrida S, Maiorano E, Mazzarol G, Pruneri G, Paganelli G, et al. Predicting the status of axillary sentinel lymph nodes in 4351 patients with invasive breast carcinoma treated in a single institution. Cancer. 2005;103: 492-500.

16. Gann PH, Colilla SA, Gapstur SM, Winchester DJ, Winchester DP. Factors associated with axillary lymph node metastasis from breast carcinoma: descriptive and predictive analyses. Cancer. 1999;86:1511-9.

17. Xie $F$, Yang $H$, Wang $S$, Zhou B, Tong F, Yang D, et al. A logistic regression model for predicting axillary lymph node metastases in early breast carcinoma patients. Sensors (Basel). 2012;12:9936-50.

18. Zhang J, Li X, Huang R, Feng WL, Kong YN, Xu F, et al. A nomogram to predict the probability of axillary lymph node metastasis in female patients with breast cancer in China: a nationwide, multicenter, 10-year epidemiological study. Oncotarget. 2017;8:35311-25.

19. Dihge L, Bendahl PO, Ryden L. Nomograms for preoperative prediction of axillary nodal status in breast cancer. Br J Surg. 2017;104:1494-505.

20. Alran S, De Rycke Y, Fourchotte V, Charitansky H, Laki F, Falcou MC, et al. Validation and limitations of use of a breast cancer nomogram predicting the likelihood of non-sentinel node involvement after positive sentinel node biopsy. Ann Surg Oncol. 2007;14:2195-201.

21. Smidt ML, Kuster DM, van der Wilt GJ, Thunnissen FB, Van Zee KJ, Strobbe $\sqcup$. Can the Memorial Sloan-Kettering Cancer Center nomogram predict the likelihood of nonsentinel lymph node metastases in breast cancer patients in the Netherlands? Ann Surg Oncol. 2005;12:1066-72.

22. Ponzone R, Maggiorotto F, Mariani L, Jacomuzzi ME, Magistris A, Mininanni $P$, et al. Comparison of two models for the prediction of nonsentinel node metastases in breast cancer. Am J Surg. 2007;193:686-92.

23. Lambert LA, Ayers GD, Hwang RF, Hunt KK, Ross MI, Kuerer HM, et al. Validation of a breast cancer nomogram for predicting nonsentinel lymph node metastases after a positive sentinel node biopsy. Ann Surg Oncol. 2006;13:310-20

24. Wolff AC, Hammond ME, Schwartz JN, Hagerty KL, Allred DC, Cote RJ, et al. American Society of Clinical Oncology/College of American Pathologists guideline recommendations for human epidermal growth factor receptor 2 testing in breast cancer. Arch Pathol Lab Med. 2007;131:18-43.

25. Hammond ME, Hayes DF, Dowsett M, Allred DC, Hagerty KL, Badve S, et al. American Society of Clinical Oncology/College of American Pathologists guideline recommendations for immunohistochemical testing of estrogen and progesterone receptors in breast cancer (unabridged version). Arch Pathol Lab Med. 2010;134:e48-72.

26. Perez EA, Dueck AC, McCullough AE, Reinholz MM, Tenner KS, Davidson NE, et al. Predictability of adjuvant trastuzumab benefit in N9831 patients using the ASCO/CAP HER2-positivity criteria. J Natl Cancer Inst. 2012;104:159-62.

27. Kim T, Giuliano AE, Lyman GH. Lymphatic mapping and sentinel lymph node biopsy in early-stage breast carcinoma: a metaanalysis. Cancer. 2006; 106:4-16.

28. Veronesi U, Paganelli G, Galimberti V, Viale G, Zurrida S, Bedoni M, et al. Sentinel-node biopsy to avoid axillary dissection in breast cancer with clinically negative lymph-nodes. Lancet. 1997;349:1864-7.

29. Krag D, Weaver D, Ashikaga T, Moffat F, Klimberg VS, Shriver C, et al. The sentinel node in breast cancer--a multicenter validation study. N Engl J Med. 1998;339:941-6.

30. Angarita FA, Elmi M, Zhang Y, Look Hong NJ. Patient-reported factors influencing the treatment decision-making process of older women with non-metastatic breast cancer: a systematic review of qualitative evidence. Breast Cancer Res Treat. 2018;171:545-64.

31. Lodi M, Scheer L, Reix N, Heitz D, Carin AJ, Thiebaut N, et al. Breast cancer in elderly women and altered clinico-pathological characteristics: a systematic review. Breast Cancer Res Treat. 2017;166:657-68.

32. Cetintas SK, Kurt M, Ozkan L, Engin K, Gokgoz S, Tasdelen I. Factors influencing axillary node metastasis in breast cancer. Tumori. 2006;92: 416-22.

33. Liu M, Wang S, Pan L, Yang D, Xie F, Liu P, et al. A new model for predicting non-sentinel lymph node status in Chinese sentinel lymph node positive breast cancer patients. PLoS One. 2014;9:e104117.

34. Kullberg C, Selander J, Albin M, Borgquist S, Manjer J, Gustavsson P. Female white-collar workers remain at higher risk of breast cancer after adjustments for individual risk factors related to reproduction and lifestyle. Occup Environ Med. 2017:74:652-8.

35. Villeneuve S, Fevotte J, Anger A, Truong T, Lamkarkach F, Gaye O, et al. Breast cancer risk by occupation and industry: analysis of the CECILE study, a population-based case-control study in France. Am J Ind Med. 2011;54: 499-509.

36. Pudrovska T, Carr D, McFarland M, Collins C. Higher-status occupations and breast cancer: a life-course stress approach. Soc Sci Med. 2013;89:53-61.

37. Smith EC, Ziogas A, Anton-Culver H. Delay in surgical treatment and survival after breast cancer diagnosis in young women by race/ethnicity. JAMA Surg. 2013;148:516-23

38. Kothari A, Fentiman IS. Diagnostic delays in breast cancer and impact on survival. Int J Clin Pract. 2003;57:200-3.

\section{Publisher's Note}

Springer Nature remains neutral with regard to jurisdictional claims in published maps and institutional affiliations.

Ready to submit your research? Choose BMC and benefit from:

- fast, convenient online submission

- thorough peer review by experienced researchers in your field

- rapid publication on acceptance

- support for research data, including large and complex data types

- gold Open Access which fosters wider collaboration and increased citations

- maximum visibility for your research: over $100 \mathrm{M}$ website views per year

At $\mathrm{BMC}$, research is always in progress.

Learn more biomedcentral.com/submissions 